\title{
Indium(III) Chloride Mediated Michael Addition of Indoles to Ketene S,S-Acetals: Synthesis of Bis- and Tris-indolylketones
}

\author{
Thokchom Prasanta Singh, Ruhima Khan, Young Ri Noh, ${ }^{\dagger}$ Sang-Gyeong Lee, ${ }^{\dagger, *}$ and Okram Mukherjee Singh ${ }^{*}$ \\ Department of Chemistry, Manipur University, Canchipur-795 003. Manipur, India. *E-mail: ok_mukherjee@yahoo.co.in \\ 'Department of Chemistry, Research Institute of Natural Science (RINS), Graduate School for Molecular Materials and \\ Nanochemistry, Gyeongsang National University, Jinju 660-701, Korea. E-mail: leesang@gnu.ac.kr \\ Received April 11, 2014, Accepted June 10, 2014
}

\begin{abstract}
A series of bis and tris-indolylketones and meridianin alkaloids are prepared by one pot Michael reaction of
\end{abstract} indole and ketene $S, S$-acetals under solvent-free condition using mild Lewis acid $\mathrm{InCl}_{3}$.

Key Words : Bis-indolylketones, Tris-indolylketones, One-pot, Solvent-free, Michael reaction

\section{Introduction}

The indole nucleus is an important structural motif in medicinal chemistry. ${ }^{1}$ Several substituted indoles have been referred to as privileged structures as they are capable of binding to many receptors with high affinity. ${ }^{2}$ Among the derivatives of indoles, bisindole scaffolds were received much attention as important building blocks for the synthesis of many natural products and other biologically active compounds. ${ }^{3}$ They possess antitumor (I), ${ }^{4}$ genotoxicity (II), ${ }^{5}$ antihyperlipidemic and antiobesity (III) ${ }^{6}$ (Figure 1) and radical scavenging activities. ${ }^{7}$ Further, tris-indolyl scaffolds are known to show bacterial metabolic ${ }^{8}$ and cytotoxic agents (IV). ${ }^{9}$

Owing to their important biological activities, many synthetic chemists are giving great attention towards the development of convenient methods for the synthesis of new indole derivatives. ${ }^{10}$ Recently, we have reported the synthesis of tetracyclic $[6,5,5,6]$ indole ring via a tandem cycloannulation of $\beta$-oxodithioester with tryptamine in onepot catalyzed by In/TFA. ${ }^{11}$ And, our literature survey revealed that ethyl-substituted ketene dithioacetals have been utilized in Michael addition reactions with indoles catalyzed by triflouroacetic acid (TFA) ${ }^{12}$ or $\mathrm{FeCl}_{3},{ }^{13}$ in presence of dichloromethane (DCM) as solvent. Thus, we were intrigued to examine the feasibility and more ecofriendly of above reported works using well known $\alpha$-oxoketene dithioacetals. ${ }^{14}$ Therefore, as a continuous interest in the development of new methodologies for the synthesis of nitrogen containing heterocyclic compounds, ${ }^{15 a-d}$ we endeavored to develop an<smiles>c1ccc2c(Cc3c[nH]c4ccccc34)c[nH]c2c1</smiles><smiles>CCCC(=O)C1Cc2cc(C(c3c[nH]c4ccccc34)c3c[nH]c4ccccc34)c3ccccc3c2OC1=O</smiles>

Antiobesity (III)

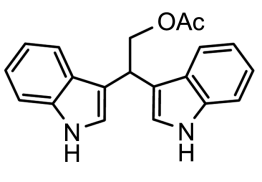

Genotoxicity (II)

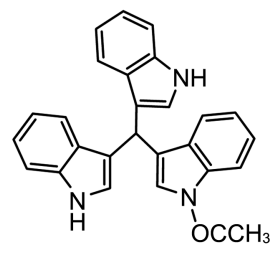

Cytotoxic activity (IV)
Figure 1. Selected bis- and tris-indole alkaloids.<smiles>[R]C(C)CC(=O)CC([R3])C([R3])CC(=O)C=C(c1cn([R2])c2ccccc12)c1cn([R2])c2ccccc12</smiles><smiles>[R2]n1ccc2ccccc21</smiles><smiles>[R]C=CC(=O)C=C([As])[Sn]</smiles><smiles>[R]C=CCCC</smiles><smiles>[R]C(=O)C=C(C)SC</smiles><smiles>[R2]N(C)c1cccc2cc(CCCCC)n([R2])c12</smiles><smiles>[R]C(=O)C=C(c1cn([R2])c2ccccc12)c1cn([R2])c2ccccc12</smiles><smiles>[R]c1cc(-c2cn([R2])c3ccccc23)nc(N)n1</smiles>

8<smiles>N=C(N)N[N+](=O)[O-]</smiles><smiles>[R]C(=O)/C=C(/SCC)c1cn([R2])c2ccccc12</smiles>

Scheme 1. Synthesis of bis, tris-indolylketones and meridinian derivatives. 
efficient, selective and mild method for the preparation of $\beta, \beta$-bisindolyl-ketones $\mathbf{4}$, tris-indolylketones 7 and meridianin derivatives 8 by treating $\alpha$-oxoketene dithioacetals with indole in the presence of $\mathrm{InCl}_{3}$ under solvent-free conditions (Scheme 1).

Initially, we expected the Michael reaction of 3,3-bis(methylthio)-1-phenylprop-2-en-1-one 1a $(1.0 \mathrm{mmol})$ and $\mathrm{N}$-methylindole 2a $(2.0 \mathrm{mmol})$ in presence of $5.0 \mathrm{~mol} \%$ of $\mathrm{InCl}_{3}$ in $\mathrm{EtOH}(5 \mathrm{~mL})$ gave the $(E)-3-(1-$ methyl- $1 H$-indol-3yl)-3-(methylthio)-1-pheny-1-prop-2-en-1-one (4a) in 65\% of yield under refluxing condition (Table 1, entry 1). So other acids such as TFA, $\mathrm{BF}_{3} \cdot \mathrm{OEt}_{2}$ and $\mathrm{FeCl}_{3}$ were also investigated, and found that these acids could not catalyzed this reaction efficiently (entries 2-4). TFA facilitated $\beta$ indolylketones formation in moderate yield of $60 \%$ (entry 2). However, we choose $\mathrm{InCl}_{3}$ over TFA, as it is a versatile stable acidic reagent with relatively mild nature and environmentally friendly in compare to TFA, which is a noxious reagent. The model reaction was performed in other solvents, such as $\mathrm{MeOH}$, DCM, and DMF but the corresponding products were obtained in only $35 \%, 60 \%$ and $56 \%$ yields, respectively (entries 5-7). To our surprise, when the reaction was performed in solvent-free condition using $\mathrm{InCl}_{3}$ (entry 8 ), the reaction gave the product in good yield of $85 \%$. Further, the catalytic loading of $\mathrm{InCl}_{3}$ was tested (entry 9), the results showed $5 \mathrm{~mol} \%$ of $\mathrm{InCl}_{3}$ was the best amount. It is concluded that the optimum reaction condition was $\mathrm{InCl}_{3}$ $(5.0 \mathrm{~mol} \%)$ as a catalyst without any solvent at $80^{\circ} \mathrm{C}$.

Having established the optimal reaction conditions, we tested scope of the substrates and found all reactions of various $\alpha$-oxoketene dithioacetals and indoles leading to corresponding 3,3-bis(1-methyl-1H-indol-3-yl) derivatives 4 (Table 2). The results of reactions of various $\alpha$-oxoketene dithioacetals with indole $\mathbf{2 a} / \mathbf{b}$ showed that the process could tolerate both aromatic ketones with electronically different

Table 1. Optimization of reaction conditions for the synthesis of $\beta$ indolylketones $^{a}$

\begin{tabular}{|c|c|c|c|c|}
\hline \multicolumn{2}{|c|}{$1 a$} & $2 a$ & $4 a$ & \\
\hline Entry & Solvent & $\begin{array}{c}\text { Catalyst } \\
\text { (equiv) }\end{array}$ & $\begin{array}{l}\text { Time } \\
\text { (hr) }\end{array}$ & $\begin{array}{c}\text { Yield }^{b} \\
(\%)\end{array}$ \\
\hline 1 & EtOH & $\mathrm{InCl}_{3}(5 \mathrm{~mol} \%)$ & 3 & 65 \\
\hline 2 & $\mathrm{EtOH}$ & TFA (5 mol \%) & 3 & 60 \\
\hline 3 & $\mathrm{EtOH}$ & $\mathrm{BF}_{3} \cdot \mathrm{OEt}_{2}(5 \mathrm{~mol} \%)$ & 3 & 45 \\
\hline 4 & EtOH & $\mathrm{FeCl}_{3}(5 \mathrm{~mol} \%)$ & 3 & 50 \\
\hline 5 & $\mathrm{MeOH}$ & $\mathrm{InCl}_{3}(5 \mathrm{~mol} \%)$ & 3 & 35 \\
\hline 6 & $\mathrm{DCM}$ & $\mathrm{InCl}_{3}(5 \mathrm{~mol} \%)$ & 3 & 60 \\
\hline 7 & DMF & $\mathrm{InCl}_{3}(5 \mathrm{~mol} \%)$ & 3 & 56 \\
\hline 8 & Solvent free & $\mathrm{InCl}_{3}(5 \mathrm{~mol} \%)$ & 2 & 85 \\
\hline 9 & Solvent free & $\mathrm{InCl}_{3}(10 \mathrm{~mol} \%)$ & 2 & 85 \\
\hline
\end{tabular}

${ }^{a}$ Reaction conditions: 1a $(1.0 \mathrm{mmol}), \mathbf{2 a}(2.0 \mathrm{mmol})$ and ${ }^{b}$ Isolated yield.
Table 2. Synthesis of bis-indolylketones ${ }^{a}$

\begin{tabular}{|c|c|c|c|c|c|}
\hline $1 \mathrm{a}-\mathrm{h}$ & & a-b & & 4a-k & \\
\hline Entry & $\mathrm{R}_{1}$ & $\mathrm{R}_{2}$ & Product & $\begin{array}{l}\mathrm{mp} \\
\left({ }^{\circ} \mathrm{C}\right)\end{array}$ & $\begin{array}{c}\text { Yield }^{c} \\
(\%)\end{array}$ \\
\hline 1 & $\mathrm{Ph}$ & $\mathrm{Me}$ & $4 a$ & $\begin{array}{c}167-169 \\
(162-164)^{b}\end{array}$ & 85 \\
\hline 2 & $\mathrm{Ph}$ & $\mathrm{H}$ & $4 b$ & $\begin{array}{c}233-235 \\
(236-238)^{b}\end{array}$ & 75 \\
\hline 3 & $\mathrm{Me}$ & $\mathrm{Me}$ & $4 c$ & $\begin{array}{c}177-179 \\
(233-235)^{b}\end{array}$ & 85 \\
\hline 4 & $\mathrm{Me}$ & $\mathrm{H}$ & $4 d$ & $223-225$ & 73 \\
\hline 5 & 4-MeO-Ph & $\mathrm{Me}$ & $4 e$ & $\begin{array}{c}170-172 \\
(165-167)^{b}\end{array}$ & 84 \\
\hline 6 & 4-MeO-Ph & $\mathrm{H}$ & $4 f$ & $\begin{array}{c}136-138 \\
(135-136)^{b}\end{array}$ & 75 \\
\hline 7 & 4-Me-Ph & $\mathrm{Me}$ & $4 \mathrm{~g}$ & $155-157$ & 83 \\
\hline 8 & 4-Cl-Ph & $\mathrm{Me}$ & $4 h$ & 206-208 & 90 \\
\hline 9 & 4-Br-Ph & $\mathrm{Me}$ & $4 i$ & $\begin{array}{c}216-218 \\
(218-220)^{b}\end{array}$ & 87 \\
\hline 10 & thionyl & $\mathrm{Me}$ & $4 j$ & $\begin{array}{c}194-196 \\
(190-192)^{b}\end{array}$ & 83 \\
\hline 11 & furyl & $\mathrm{Me}$ & $4 k$ & $165-167$ & 82 \\
\hline
\end{tabular}

${ }^{a}$ Reaction conditions: 1 (2.0 $\left.\mathrm{mmol}\right), 2(4.0 \mathrm{mmol})$, catalyst $(5.0 \mathrm{~mol} \%)$. ${ }^{b}$ Literature mp. (ref. 12-13); solvent-free, $80{ }^{\circ} \mathrm{C}, 2 \mathrm{~h}$. ${ }^{c}$ Isolated yield.

substituents (entries 5-9) and even extremely electron-rich aromatic $\alpha$-oxoketene dithioacetals (such as 2-acetyl furan and 2-acetyl thiophene) (entries 10-11), and even aliphatic ketones such as methyl (entries 3-4). It is observed that the substituents on the aromatic rings had some influence on the yields of products 4 . The aromatic ketones with electronwithdrawing groups, such as chloro and bromo (entries 8-9) reacted faster and gave higher yields than those with electrondonating groups, such as methyl and methoxyl groups (entries 5-7). The $N$-methylated indole afforded higher yields than indole, which should be related to the electronic effect.

Then, the $\alpha$-oxoketene dithioacetal $\mathbf{1 b}$ was next subjected to condensation with aromatic aldehyde $\mathbf{5}$ in the presence of $5 \%$ alcoholic $\mathrm{KOH}$ and ethanol to give the cinnamoylketene dithioacetals 6. It was anticipated that $\alpha$-cinnamoylketene dithioacetals 6 would undergo 1,4-addition with indole (ratio 1:3) with subsequent elimination of the two-SMe groups and further addition of one indole (2a) to double bond conjugate to carbonyl group would yield the tris-indolylketones. Thus, cinnamoylketene dithio-acetals $(6 \mathbf{6} ; 2.0 \mathrm{mmol})$ and indole (2a; $6.0 \mathrm{mmol}$ ) were stirred for $2.5 \mathrm{~h}$ under solvent-free condition at $100{ }^{\circ} \mathrm{C}$ using $\mathrm{InCl}_{3}(5.0 \mathrm{~mol} \%)$, as expected, the product 1,1,5-tris(1-methyl-1 $H$-indol-3-yl)-5-phenylpent-1en-3-one (7a) was obtained in good yield (78\%). The longer in reaction time and higher temperature may be attributed 
Table 3. Synthesis of tris-indolylketones

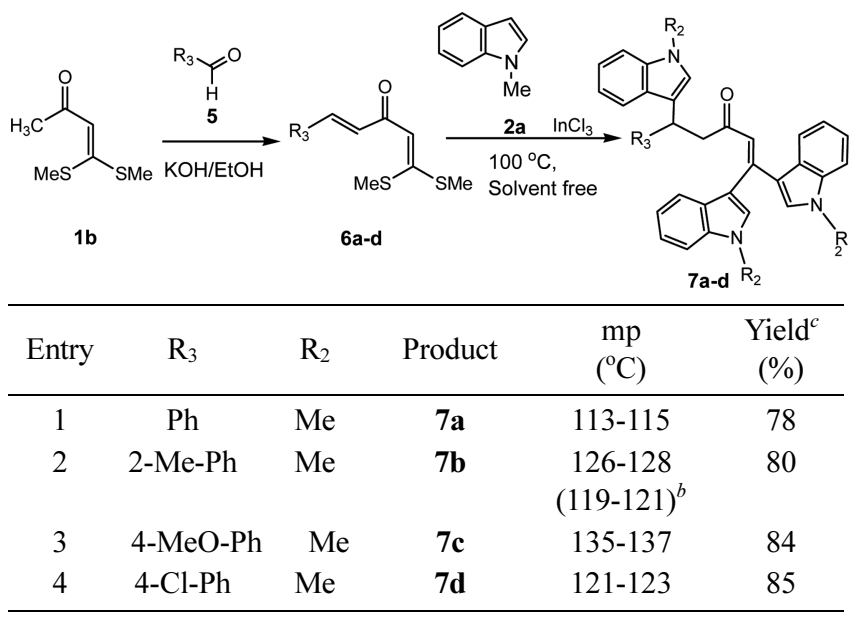

Reaction conditions: $6(2.0 \mathrm{mmol}), \mathbf{2}(6.0 \mathrm{mmol})$, catalyst $(5.0 \mathrm{~mol} \%)$. ${ }^{b}$ Literature mp. (ref. 13); solvent-free, $100{ }^{\circ} \mathrm{C}, 2.5 \mathrm{~h}$. ${ }^{c}$ Isolated yield.

due to addition of three indole moieties to $\mathbf{6}$ as compare to $\mathbf{1}$, which accommodate only two indole moieties.

Further, we wished to synthesize meridianin alkaloids as they are biologically important. Thus, when 1a $(1.0 \mathrm{mmol})$ and $N$-methylindole $(1.0 \mathrm{mmol})$ were reacted in presence of $5.0 \mathrm{~mol} \%$ of $\mathrm{InCl}_{3}$, the $\beta$-indolylketones 3 was obtained in poor yield of $45 \%$. In next experiment, to the reaction mixture of 1a and $\mathbf{2 a}$, guanidine nitrate $(0.5 \mathrm{mmol})$ and $\mathrm{KOH}(1.25 \mathrm{mmol})$ were added and refluxed in $\mathrm{EtOH}(5 \mathrm{~mL})$ for $18 \mathrm{~h}$ and determined by TLC giving the expected product in good yield of $79 \%$. Thus, four derivatives of meridianin alkaloids were synthesized by using the same procedure (Table 3).

The diversity of this protocol with respect to $\alpha$-oxoketene dithioacetals 1a-h (Table 2), cinnamoylketene dithioacetals 6a-d (Table 3 ) and synthesis of meridianin alkaloids (Table 4) are well represented following the environmentally

Table 4. Synthesis of meridianin alkaloids

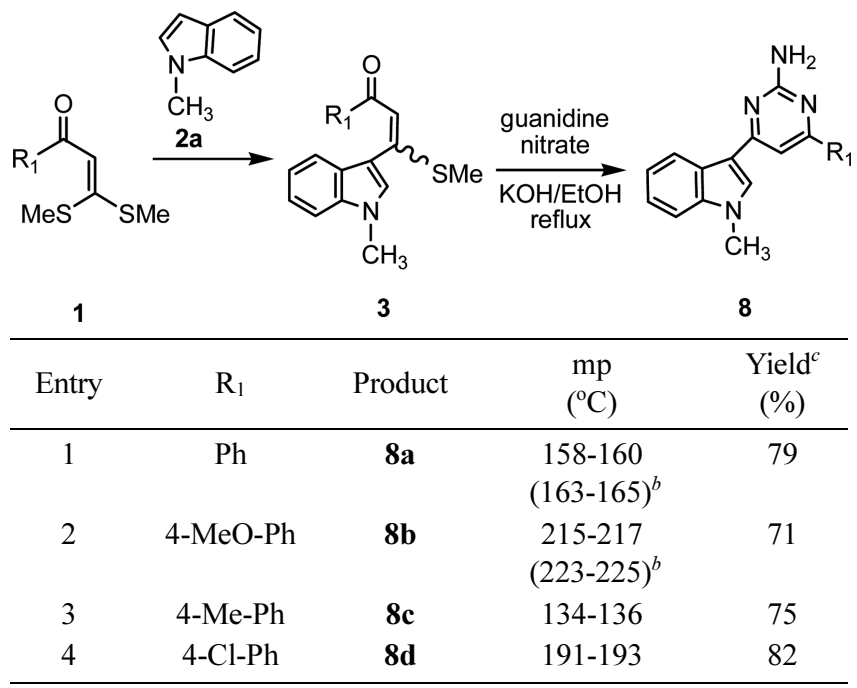

${ }^{a}$ Reaction conditions: $1(2.0 \mathrm{mmol}), \mathbf{2}(2.0 \mathrm{mmol}) .{ }^{b}$ Literature mp. (ref. 16). ${ }^{c}$ Isolated yield.

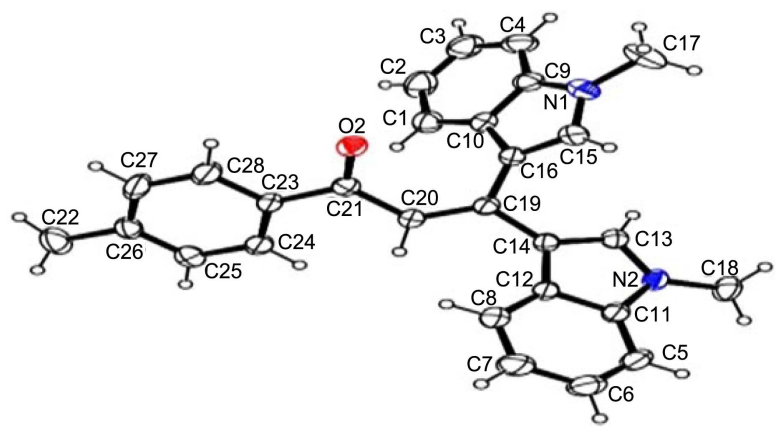

Figure 2. ORTEP diagram of $\mathbf{4 g}$ with ellipsoids at $30 \%$ probability.

benign process. The structures of all the newly synthesized compounds 4a-l, 7a-d and 8a-d were confirmed satisfactory from their elemental and spectral (IR, ${ }^{1} \mathrm{H}$ and ${ }^{13} \mathrm{C} \mathrm{NMR}$ ) studies and also comparing to the known compounds. X-Ray diffraction analysis of $\beta, \beta$-indolylketone $\mathbf{4 g}$ further supports the structural elucidation (Figure 2).

\section{Experimental}

All compounds were fully characterized by spectroscopic data. ${ }^{1} \mathrm{H}$ NMR (300 MHz) and ${ }^{13} \mathrm{C}$ NMR (75 MHz) spectra were recorded on FT-NMR spectrometer using $\mathrm{CDCl}_{3}$. Chemical shifts $\delta$ are in parts per million (ppm) with either $\mathrm{CDCl}_{3}$ as solvent and are relative to tetramethylsilane (TMS) as the internal reference. Data are reported as follows: chemical shift, multiplicity $(\mathrm{s}=$ singlet, $\mathrm{d}=$ doublet, $\mathrm{t}=$ triplet, $\mathrm{m}=$ multiplet, $\mathrm{br}=$ broad) and coupling constants $(J)$ in Hertz. The FT-IR spectra were recorded on a FT-IR spectrometer (KBr). Gas chromatography-electron impact mass spectrometry (GC-EIMS) spectra were measured on a Varian spectrometer using ionization by fast atom bombardment (FAB). Melting points were determined on a "Veego" capillary melting point apparatus and are uncorrected. Silica gel 60 was used for column separations. Chemical yields refer to the pure isolated substances.

Typical Procedure for Bis-indolyl Synthesis. The $\alpha$-охоketene dithioacetal $(2.0 \mathrm{mmol})$ and indole $(4.0 \mathrm{mmol})$ were mixed throughly to get a paste like mixture. $\operatorname{InCl}_{3}(5 \mathrm{~mol} \%)$ was added to the pasty mixture, which was then stirred at 80 ${ }^{\circ} \mathrm{C}$ for the stipulated period of time. After completion of the reaction (as monitored by TLC), $\mathrm{CH}_{2} \mathrm{Cl}_{2}(10 \mathrm{~mL})$ was added to the mixture and then $20 \mathrm{~mL}$ of $\mathrm{H}_{2} \mathrm{O}$ was poured to the mixture. The organic layer was dried over anhydrous $\mathrm{Na}_{2} \mathrm{~S}_{3}$ and the solvent was evaporated under reduced pressure and purification by column chromatography over silica gel, eluting with ethyl acetate-hexane $(2: 8, \mathrm{v} / \mathrm{v})$, to give a yellow solid with $83 \%$ yield.

3,3-Bis(1-methyl-1 $\boldsymbol{H}$-indol-3-yl)-1-p-tolylprop-2-en-1-one (4g): Yellow solid; mp 155-157 ${ }^{\circ} \mathrm{C}$; ${ }^{1} \mathrm{H}$ NMR $(400 \mathrm{MHz}$, $\left.\mathrm{CDCl}_{3}\right) \delta 7.92$ and 7.82, (s each, $\left.1: 1 \mathrm{H}, \mathrm{ArH}\right), 7.38(\mathrm{~d}, J=8.4$ $\mathrm{Hz}, 1 \mathrm{H}, \mathrm{ArH}), 7.26-7.32$ (m, 2H, ArH), 7.17-7.23 (m, 6H, $\mathrm{ArH}), 7.12(\mathrm{~s}, 1 \mathrm{H},-\mathrm{C}=\mathrm{CH}), 6.97$ (t, $J=14.8 \mathrm{~Hz}, 1 \mathrm{H}, \mathrm{ArH})$, $6.79(\mathrm{~d}, J=8.0 \mathrm{~Hz}, 1 \mathrm{H}, \mathrm{ArH}), 3.78$ and 3.76 (s each, $3: 3 \mathrm{H}$, $\left.2 \mathrm{NCH}_{3}\right), 2.17\left(\mathrm{~s}, 3 \mathrm{H}, \mathrm{CH}_{3}\right) ;{ }^{13} \mathrm{C} \mathrm{NMR}\left(150 \mathrm{MHz}, \mathrm{CDCl}_{3}\right) \delta$ 
$191.2,143.6,141.9,138.0,137.7,137.0,133.5,132.5,128.7$, $128.3,127.4,126.5,122.6,121.9,121.3,121.1,120.9,120.0$, 118.6, 117.7, 115.0, 110.0, 109.4, 33.3, 33.1, 21.6; IR (KBr) 3062, 2980, 1625, 1386, $1141 \mathrm{~cm}^{-1}$; MS m/z $404(\mathrm{M})^{+}$; Calcd for $\mathrm{C}_{28} \mathrm{H}_{24} \mathrm{~N}_{2} \mathrm{O}$ : C, 83.14; H, 5.98; N, 6.93; O, 3.96 . Found: C, 83.06; H, 5.86; N, 6.85; O, 3.85.

1-(4-Chlorophenyl)-3,3-bis(1-methyl-1 $\boldsymbol{H}$-indol-3-yl)prop2-en-1-one (4h): Yellow solid; mp 206-208 ${ }^{\circ} \mathrm{C}$; ${ }^{1} \mathrm{H}$ NMR $\left(400 \mathrm{MHz}, \mathrm{CDCl}_{3}\right) \delta 7.95$ and 7.76 (d each, $J=8.4$ and 7.4 $\mathrm{Hz}, 1: 2 \mathrm{H}, \mathrm{ArH}), 7.40$ (d, $J=7.4 \mathrm{~Hz}, 1 \mathrm{H}, \mathrm{ArH}), 7.34-7.38$ (m, 1H, ArH), 7.31-7.33 (m, 2H, ArH), 7.22-7.30 (m, 7H, $\mathrm{ArH}), 7.18(\mathrm{~s}, 1 \mathrm{H},-\mathrm{C}=\mathrm{CH}), 7.01(\mathrm{t}, J=14.0 \mathrm{~Hz}, 1 \mathrm{H}, \mathrm{ArH})$, 3.77 and 3.75 (s each, $\left.3: 3 \mathrm{H}, 2 \mathrm{NCH}_{3}\right) ;{ }^{13} \mathrm{C} \mathrm{NMR} \mathrm{(150} \mathrm{MHz,}$ $\left.\mathrm{CDCl}_{3}\right) \delta 190.8,148.2,144.3,138.0,137.0,133.9,133.1$, $131.7,130.1,127.8,127.7,126.6,122.7,121.9,121.4,121.1$, 121.0, 120.0, 118.7, 116.0, 114.3, 110.0, 109.5, 33.2 (2C); IR (KBr) 3063, 2952, 1624, 1379, $1125 \mathrm{~cm}^{-1}$; MS m/z 424 $(\mathrm{M})^{+}$; Calcd for $\mathrm{C}_{27} \mathrm{H}_{21} \mathrm{ClN}_{2} \mathrm{O}$ : C, 76.32; H, 4.98; Cl, 8.34; N, 6.59; O, 3.77. Found: C, 76.27; H, 4.88; Cl, 8.29; N, 6.50; $\mathrm{O}, 3.71$.

1-(Furan-2-yl)-3,3-bis(1-methyl-1H-indol-3-yl)prop-2en-1-one (4k): Pink solid; mp 165-167 ${ }^{\circ} \mathrm{C} ;{ }^{1} \mathrm{H}$ NMR (400 $\mathrm{MHz}, \mathrm{CDCl}_{3}$ ) $\delta 7.95$ and 7.77, (d each, $J=8.4$ and $7.4 \mathrm{~Hz}$, 1:1H, ArH), 7.47 and 7.50 (s each, 1:1 H, ArH), 7.18-7.42 (m, 8H, ArH), 7.16-7.13 (m, 1H, ArH), 6.98-7.12 (m, 1H, ArH), 3.81 and 3.75 (s each, $\left.3: 3 \mathrm{H}, 2 \mathrm{NCH}_{3}\right) ;{ }^{13} \mathrm{C} \mathrm{NMR}(150$ $\left.\mathrm{MHz}, \mathrm{CDCl}_{3}\right) \delta 180.2,149.2,148.4,147.3,143.0,141.4$, $132.4,131.3,131.0,130.2,129.7,129.2,128.8,128.7,128.6$, $127.0,126.7,124.7,123.3,117.5,115.9,113.6,110.0,30.9$, 30.5; IR (KBr) 3046, 2945, 1624, 1512, $1120 \mathrm{~cm}^{-1}$; MS $\mathrm{m} / z$ $380(\mathrm{M})^{+}$; Calcd for $\mathrm{C}_{25} \mathrm{H}_{20} \mathrm{~N}_{2} \mathrm{O}_{2}$ : C, 78.93; H, 5.30; N, 7.36; O, 8.41. Found: C, 78.87; H, 5.23; N, 7.29; O, 8.36.

Typical Procedure for Tris-indolyl Synthesis. The cinnamoylketene dithioacetals $\mathbf{6 a}(2.0 \mathrm{mmol})$ and indole $\mathbf{2 a}(6.0$ mmol) were mixed throughly to get a paste like mixture. $\mathrm{InCl}_{3}(5 \mathrm{~mol} \%)$ was added to the pasty mixture, which was then stirred at $100{ }^{\circ} \mathrm{C}$ for the stipulated period of time. After completion of the reaction (as monitored by TLC), $\mathrm{CH}_{2} \mathrm{Cl}_{2}$ $\left(15 \mathrm{~mL}\right.$ ) was added to the mixture and then $30 \mathrm{~mL}$ of $\mathrm{H}_{2} \mathrm{O}$ was poured to the mixture. The organic layer was dried over anhydrous $\mathrm{Na}_{2} \mathrm{~S}_{3}$ and the solvent was evaporated under reduced pressure and purification by column chromatography over silica gel, eluting with acetate-hexane $(2: 8, \mathrm{v} / \mathrm{v})$, to give a yellow solid with $78 \%$ yield.

1,1,5-Tris(1-methyl-1H-indol-3-yl)-5-phenylpent-1-en3-one (7a): Yellow solid; mp 113-115 ${ }^{\circ} \mathrm{C}$; ${ }^{1} \mathrm{H}$ NMR (400 $\left.\mathrm{MHz} \mathrm{CDCl}_{3}\right) \delta 7.83(\mathrm{~d}, J=8.0 \mathrm{~Hz}, 1 \mathrm{H}, \mathrm{ArH}), 7.36-7.41(\mathrm{~m}$, $6 \mathrm{H}, \mathrm{ArH}), 7.35-7.21$ (m, 10H, ArH), 7.14-7.15 (m, 2H, ArH), $7.01(\mathrm{~s}, 1 \mathrm{H}, \mathrm{ArH}), 6.80(\mathrm{~d}, J=7.2 \mathrm{~Hz}, 2 \mathrm{H}, \mathrm{ArH}), 4.42$ (t, $J=$ $14.0 \mathrm{~Hz}, 1 \mathrm{H}, \mathrm{CH}), 3.81\left(\mathrm{~s}, 3 \mathrm{H}, \mathrm{NCH}_{3}\right), 3.76$ and $3.74(\mathrm{~s}$ each, $\left.6 \mathrm{H}, 2 \mathrm{NCH}_{3}\right), 3.21-3.23\left(\mathrm{~m}, 2 \mathrm{H}, \mathrm{CH}_{2}\right) ;{ }^{13} \mathrm{C} \mathrm{NMR}(150$ $\left.\mathrm{MHz}, \mathrm{CDCl}_{3}\right) \delta 200.0,142.4,142.0,139.2,137.6,137.3$, 134.6, 132.4, 131.3, 131.1, 131.1, 129.9, 129.4, 129.4, 129.0, $128.4,128.3,128.1,127.3,127.1,126.9,124.8,124.6,124.3$, $123.9,123.5,123.1,122.8,120.5,118.1,117.5,112.9,49.1$, 41.4, 33.6, 33.3, 33.1; IR (KBr) 3071, 2966, 1614, 1377, $1124 \mathrm{~cm}^{-1}$; MS m/z $547(\mathrm{M})^{+}$; Calcd for $\mathrm{C}_{38} \mathrm{H}_{33} \mathrm{~N}_{3} \mathrm{O}: \mathrm{C}$,
83.33; H, 6.07; N, 7.67; O, 2.92; Found: C, 83.25; H, 5.97; N, 7.62; O, 2.86.

5-(4-Methoxyphenyl)-1,1,5-tris(1-methyl-1H-indol-3yl)pent-1-en-3-one (7c): Pale white solid; mp $135-137{ }^{\circ} \mathrm{C}$; ${ }^{1} \mathrm{H}$ NMR $\left(400 \mathrm{MHz}, \mathrm{CDCl}_{3}\right) \delta 7.86(\mathrm{~d}, J=7.8 \mathrm{~Hz}, 1 \mathrm{H}$, ArH), 7.39-7.48 (m, 6H, ArH), 7.21-7.37 (m, 9H, ArH), 7.18-7.20 (m, 2H, ArH), $7.01(\mathrm{~s}, 1 \mathrm{H}, \mathrm{ArH}), 6.79(\mathrm{~s}, 1 \mathrm{H}$, ArH), 4.41 (t, $J=13.6 \mathrm{~Hz}, 1 \mathrm{H}, \mathrm{CH}), 3.91\left(\mathrm{~s}, 3 \mathrm{H}, \mathrm{OCH}_{3}\right)$, $3.75\left(\mathrm{~s}, 3 \mathrm{H}, \mathrm{NCH}_{3}\right), 3.72\left(\mathrm{~s}, 6 \mathrm{H}, 2 \mathrm{NCH}_{3}\right), 3.30-3.32(\mathrm{~m}, 2 \mathrm{H}$, $\left.\mathrm{CH}_{2}\right) ;{ }^{13} \mathrm{C} \mathrm{NMR}\left(150 \mathrm{MHz}, \mathrm{CDCl}_{3}\right) \delta 199.8,148.4,147.0$, $141.5,140.0,139.3,139.1,139.1,138.2,136.2,136.1,132.5$, $132.3,130.1,129.0,128.8,128.5,128.3,128.1,126.9,126.8$, $124.5,124.0,123.3,117.5,116.7,113.5,54.5,50.3,41.0$, $33.8,33.2,33.1$; IR (KBr) 3075, 3001, 1623, $1134 \mathrm{~cm}^{-1}$; MS $m / z 577(\mathrm{M})^{+}$; Calcd for $\mathrm{C}_{39} \mathrm{H}_{35} \mathrm{~N}_{3} \mathrm{O}_{2}: \mathrm{C}, 81.08 ; \mathrm{H}, 6.11 ; \mathrm{N}$, 7.27; O, 5.54; Found: C, 81.01; H, 6.03; N, 7.20; O, 5.47.

5-(4-Chlorophenyl)-1,1,5-tris(1-methyl-1H-indol-3-yl)pent-1-en-3-one (7d): Yellow solid; mp 121-123 ${ }^{\circ} \mathrm{C}$; ${ }^{1} \mathrm{H}$ NMR (400 MHz, $\left.\mathrm{CDCl}_{3}\right) \delta 7.56(\mathrm{~d}, J=7.6 \mathrm{~Hz}, 1 \mathrm{H}, \mathrm{ArH})$, 7.31-7.38 (m, 5H, ArH), 7.13-7.29 (m, 9H, ArH), 6.84-6.92 (m, 3H, ArH), 6.80 (d, $J=7.8 \mathrm{~Hz}, 2 \mathrm{H}, \mathrm{ArH}), 4.42$ (t, $J=$ $13.8 \mathrm{~Hz}, 1 \mathrm{H}, \mathrm{CH}), 3.73$ (s, 3H, NCH 3$), 3.69$ (s, 3H, $\left.\mathrm{NCH}_{3}\right)$, $3.68\left(\mathrm{~s}, 3 \mathrm{H}, \mathrm{NCH}_{3}\right), 3.11-3.13\left(\mathrm{~m}, 2 \mathrm{H}, \mathrm{CH}_{2}\right) ;{ }^{13} \mathrm{C} \mathrm{NMR}(150$ $\left.\mathrm{MHz}, \mathrm{CDCl}_{3}\right) \delta 199.5,142.3,142.2,142.2,141.1,138.2$, 138.1, 138.0, 134.1, 133.8, 133.6, 130.0, 130.8, 128.6, 128.4, $128.2,128.0,127.9,121.6,121.5,121.5,120.2,120.2,111.5$, $111.5,107.2,107.1,107.1,48.0,40.6,33.8,33.6,33.4$; IR (KBr) 3068, 3012, 1629, $1127 \mathrm{~cm}^{-1}$; MS m/z $581(\mathrm{M})^{+}$; Calcd for $\mathrm{C}_{38} \mathrm{H}_{32} \mathrm{ClN}_{3} \mathrm{O}$ : C, 78.40; H, 5.54; Cl, 6.09; N, 7.22; O, 2.75; Found: C, 78.32; H, 5.47; Cl, 6.01; N, 7.16; O, 2.68 .

Typical Procedure for Mono-indolyl Synthesis. The experimental procedure is same as for the synthesis of $\mathbf{4 a - k}$, only the molar ratio of $\mathbf{1 a}$ and $\mathbf{2 a}$ are in $1: 1$ ratio.

(Z)-3-(1-Methyl-1H-indol-3-yl)-3-(methylthio)-1-p-tolylprop-2-en-1-one (3a): Yellow solid; mp 108-110 ${ }^{\circ} \mathrm{C} ;{ }^{1} \mathrm{H}$ NMR (400 MHz, $\mathrm{CDCl}_{3}$ ) $\delta 7.93$ and 7.78 (d each, $J=8.0$ and $8.4 \mathrm{~Hz}, 1: 2 \mathrm{H}, \mathrm{ArH}), 7.27-7.41$ (m, 2H, ArH), 7.19-7.24 $(\mathrm{m}, 5 \mathrm{H}, \mathrm{ArH}), 6.96(\mathrm{t}, J=14.3 \mathrm{~Hz}, 1 \mathrm{H}, \mathrm{ArH}), 3.75(\mathrm{~s}, 3 \mathrm{H}$, $\left.\mathrm{NCH}_{3}\right), 2.78\left(\mathrm{~s}, 3 \mathrm{H}, \mathrm{CH}_{3}\right), 2.41(\mathrm{~s}, 3 \mathrm{H}) ;{ }^{13} \mathrm{C} \mathrm{NMR}(150 \mathrm{MHz}$, $\left.\mathrm{CDCl}_{3}\right) \delta 188.9,160.4,144.4,131.2,131.1,130.2,128.9$, $128.5,128.4,128.3,126.8,126.7,124.5,123.8,123.5,117.5$, 116.3, 31.1, 20.8, 18.3; IR (KBr) 2987, 1641, 1523, 1211 $\mathrm{cm}^{-1}$; MS m/z $307(\mathrm{M})^{+}$; Calcd for $\mathrm{C}_{19} \mathrm{H}_{17} \mathrm{NOS}$ : C, 74.23; H, 5.57; N, 4.56; O, 5.20; S, 10.43; Found: C, 74.16; H, 5.51; N, 4.49; O, 5.16; S, 10.36 .

A General Procedure for Synthesis of Meridinian Derivatives 8. A mixture of $3(0.25 \mathrm{mmol})$, guanidine nitrate $(0.5$ $\mathrm{mmol})$ and $\mathrm{KOH}(1.25 \mathrm{mmol})$ were refluxed in EtOH (5 $\mathrm{mL}$ ) for $18 \mathrm{~h}$ until all the starting materials was completely consumed as indicated by TLC. The mixture was cooled to room temperature and $15 \mathrm{~mL} \mathrm{CH}_{2} \mathrm{Cl}_{2}$ was added, and the reactions mixture was then filtered. The volatiles in the filtrate were evaporated under reduced pressure and the resultant residue was purified by silica gel column chromatography (ethyl acetate/hexane 1:9, v/v) to afford $8 \mathbf{a}$ as a white solid (79\%). 
4-(1-Methyl-1 $\boldsymbol{H}$-indol-3-yl)-6-p-tolylpyrimidin-2-amine (8c): Pale white solid; mp 134-136 ${ }^{\circ} \mathrm{C} ;{ }^{1} \mathrm{H}$ NMR $(300 \mathrm{MHz}$, $\left.\mathrm{CDCl}_{3}\right) \delta 8.81(\mathrm{~d}, J=3.3 \mathrm{~Hz}, 1 \mathrm{H}, \mathrm{ArH}), 8.50$ (s, 1H, ArH), $8.21(\mathrm{~d}, J=4.2 \mathrm{~Hz}, 2 \mathrm{H}, \mathrm{ArH}), 7.76$ (d, $J=5.4 \mathrm{~Hz}, 2 \mathrm{H}, \mathrm{ArH})$, 7.45 and 7.35 (t each, $J=7.2$ and $6.3 \mathrm{~Hz}, 1: 1: 1 \mathrm{H}, \mathrm{ArH}), 6.86$ (s, 1H, ArH), $6.53\left(\mathrm{~s}, 2 \mathrm{H}, \mathrm{NH}_{2}\right), 3.72\left(\mathrm{~s}, 3 \mathrm{H}, \mathrm{NCH}_{3}\right), 2.15$ (s, $\left.3 \mathrm{H}, \mathrm{CH}_{3}\right) ;{ }^{13} \mathrm{C} \mathrm{NMR}\left(150 \mathrm{MHz}, \mathrm{CDCl}_{3}\right) \delta 161.7,158.6,156.4$, 139.1, 136.6, 135.2, 131.6, 128.0, 122.7, 122.5, 121.1, 112.5, 125.2, 111.8, 110.0, 98.1, 32.8, 19.9; IR (KBr) 3375, 1523, $1234 \mathrm{~cm}^{-1}$; MS m/z $314(\mathrm{M})^{+}$; Calcd for $\mathrm{C}_{20} \mathrm{H}_{18} \mathrm{~N}_{4}$ : C, 75.98; H, 5.37; N, 18.65; Found: C, 75.88; H, 5.30; N, 18.55 .

4-(4-Chlorophenyl)-6-(1-methyl-1H-indol-3-yl)pyrimidi n-2-amine (8d): White solid; mp 191-193 ${ }^{\circ} \mathrm{C}$; ${ }^{1} \mathrm{H}$ NMR $\left(300 \mathrm{MHz}, \mathrm{CDCl}_{3}\right) \delta 8.56(\mathrm{~d}, J=4.8 \mathrm{~Hz}, 1 \mathrm{H}, \mathrm{ArH}), 8.26(\mathrm{~s}$, $1 \mathrm{H}, \mathrm{ArH}), 8.11(\mathrm{~d}, J=4.2 \mathrm{~Hz}, 1 \mathrm{H}, \mathrm{ArH}), 7.44(\mathrm{~d}, J=5.7 \mathrm{~Hz}$, $1 \mathrm{H}, \mathrm{ArH}$ ), 7.24 and 7.03 (t each, $J=6.0$ and $3.9 \mathrm{~Hz}, 1: 1 \mathrm{H}$, ArH), $6.86(\mathrm{~s}, 1 \mathrm{H}, \mathrm{ArH}), 6.49$ (s, 1H, ArH), $3.78(\mathrm{~s}, 3 \mathrm{H}$, $\left.\mathrm{NCH}_{3}\right), 2.15\left(\mathrm{~s}, 3 \mathrm{H}, \mathrm{CH}_{3}\right) ;{ }^{13} \mathrm{C} \mathrm{NMR}\left(150 \mathrm{MHz}, \mathrm{CDCl}_{3}\right) \delta$ 161.7, 159.6, 157.5, 141.8, 135.6, 135.2, 133.0, 130.6, 129.5, $127.9,124.7,120.1,120.0,110.8,110.7,100.1,33.0$; IR (KBr) 3365, 1554, $1241 \mathrm{~cm}^{-1}$; MS m/z $334(\mathrm{M})^{+}$; Calcd for $\mathrm{C}_{19} \mathrm{H}_{15} \mathrm{ClN}_{4}$ : C, 68.16; H, 4.52; Cl, 10.59; N, 16.73; Found: C, 68.09; H, 4.47; Cl, 10.51; N, 16.64.

\section{Conclusion}

Michael addition of indoles with $\alpha$-oxoketene dithioacetal was realized by using catalytic amount of mild Lewis acid $\mathrm{InCl}_{3}$ under solvent-free conditions, affording bis \& trisindolylketones and further leading to the in-situ synthesize of meridianin alkaloids. The reaction avoids the use of toxic solvents, the overall yields of the products are good and starting materials are cheaply available in compare to ethyl-substituted dithioacetals.

Acknowledgments. This research was supported by Basic Science Research Program through the National Research Foundation of Korea (NRF) funded by the Ministry of Education, Science and Technology (Grant Number: 2010-
0023775). Thokchom Prasanta Singh and Young Ri Noh are equally contributed for this paper.

\section{References}

1. (a) Sundberg, R. J. Indoles; Academic Press: New York, USA, 1996. (b) Faulkner, D. J. Nat. Prod. Rep. 2001, 18, 1.

2. (a) Gilchrist, T. L. J. Chem. Soc. Perkin Trans. 1 2001, 20, 2491. (b) Horton, D. A.; Bourne, G. T.; Smythe, M. L. Chem. Rev. 2003, 103, 893.

3. Yang, C.-G.; Huang, H.; Jiang, B. Curr. Org. Chem. 2004, 8, 1691.

4. (a) Roy, A.; Bosedasgupta, S.; Ganguly, A.; Jaisankar, P.; Majumder, H. K. Antimicrob. Agents Chemother. 2009, 53, 2589. (b) Safe, S.; Papineni, S.; Chintharlapalli, S. Cancer Lett. 2008, 269, 326.

5. Singh, H.; Singh, K. Tetrahedron 1988, 44, 5897.

6. Sashidhara, K. V.; Kumar, M.; Sonkar, R.; Singh, B. S.; Khannaand, A. K.; Bhatia, G. J. Med. Chem. 2012, 55, 2769.

7. (a) Benabadji, S. H.; Wen, R.; Zheng, J.; Dong, X.; Yuan, S. Acta Pharmacol. Sin. 2004, 25, 666. (b) Praveen, C.; Kumar, P. D.; Muralidharan, D.; Perumal, P. T. Bioorg. Med. Chem. Lett. 2010, 24, 7292 .

8. Veluri, R.; Oka, I.; Wagner-Döbler, I.; Laatsch, H. J. Nat. Prod. 2003, 66, 1520.

9. Li, J.; Guang, B.; Wang, L.; Li, B.; Zhang, G. Heterocycles 2003, 60, 1307.

10. (a) Boyarskikh, V.; Nyong, A.; Rainier, J. D. Angew. Chem. Int. Ed. 2008, 47, 5374. (b) Cacchi, S.; Fabrizi, G. Chem. Rev. 2005, 105, 2873. (c) Cadierno, V.; Francos, J.; Gimeno, J. Chem. Commun. 2010, 4175 .

11. Singh, T. P.; Bhattarcharya, S.; Singh, O. M. Org. Lett. 2013, 15, 1974.

12. Yu, H.; Yu, Z. Angew. Chem. Int. Ed. 2009, 48, 2929.

13. Yu, H.; Li, T.; Liao, P. Synthesis 2012, 44, 3743.

14. For selected recent reports, see: (a) Pan, L.; Bi, X.; Liu. Q. Chem. Soc. Rev. 2013, 42, 1251. (b) Junjappa, H.; Ila, H.; Asokan, C. V. Tetrahedron 1990, 46, 5423. (c) Ila, H.; Junjappa, H.; Mohanta, P. K. Progress in Heterocyclic Chemistry; Gribble, G. W., Gilchrist, T. L., Eds.; Pergamon: New York, 2001; p 1. (d) Misra, N. C.; Ila, H. J. Org. Chem. 2010, 75, 5195. (e) Yadav, A. K.; Peruncheralathan, S.; Ila, H.; Junjappa, H. J. Org. Chem. 2007, 72, 1388.

15. (a) Devi, N. S.; Singh, S. J.; Singh, O. M. Synlett 2012, 23, 2111. (b) Devi, L. R.; Singh, O. M. Indian J. Chem: B 2012, 9, 1426. (c) Singh, O. M.; Devi, N. S. J. Org. Chem. 2009, 74, 3141. (d) Singh, O. M.; Singh, S. J.; Devi, M. B.; Devi, L. N.; Singh, N. I.; Lee, S. G Bioorg. Med. Chem. Lett. 2008, 18, 6462. 